\title{
Association between suicidal symptoms and repeat suicidal behaviour within a sample of hospital-treated suicide attempters
}

\author{
Derek P. de Beurs, Claudia D. van Borkulo and Rory C. O'Connor
}

\section{Background}

Suicidal behaviour is the end result of the complex relation between many factors which are biological, psychological and environmental in nature. Network analysis is a novel method that may help us better understand the complex association between different factors.

\section{Aims}

To examine the relationship between suicidal symptoms as assessed by the Beck Scale for Suicide Ideation and future suicidal behaviour in patients admitted to hospital following a suicide attempt, using network analysis.

\section{Method}

Secondary analysis was conducted on previously collected data from a sample of 366 patients who were admitted to a scottish hospital following a suicide attempt. Network models were estimated to visualise and test the association between baseline symptom network structure and suicidal behaviour at 15 -month follow-up.

\section{Results}

Network analysis showed that the desire for an active attempt was found to be the most central, strongly related suicide symptom. Of the 19 suicide symptoms that were assessed at baseline, 10 symptoms were directly related to repeat suicidal behaviour. When comparing baseline network structure of repeaters $(n=94)$ with the network of non-repeaters $(n=272)$, no significant differences were found.

\section{Conclusions}

Network analysis can help us better understand suicida behaviour by visualising the complex relation between relevant symptoms and by indicating which symptoms are most central within the network. These insights have theoretical implications as well as informing the assessment and treatment of suicidal behaviour.

\section{Declaration of interest}

None.

\section{Copyright and usage}

(C) The Royal College of Psychiatrists 2017. This is an open access article distributed under the terms of the Creative commons Non-Commercial, No Derivatives (CC BY-NC-ND) license.

A key concept in network analysis is the centrality of the Suicide is a major public health issue worldwide. ${ }^{1}$ In recent decades, epidemiological studies have identified many risk factors for suicidal behaviour, such as gender, age and mental illness. ${ }^{2}$ Despite these advances, the relationship between risk factors and suicidal behaviour is not fully understood. Suicidal behaviour remains difficult to predict ${ }^{3}$ as it is the end result of a complex relation of many factors which are biological, psychological and environmental in nature. ${ }^{4}$ To be able to more effectively understand suicidal behaviour, it is argued that we need to move beyond traditional epidemiological studies and analysis, and identify methods that can better help us understand the complexity of suicidal behaviour. ${ }^{3,5}$ One new promising approach to the conceptualisation of complex problems such as suicidal behaviour is the network perspective.

\section{Network analyses}

Within the field of psychopathology, the network perspective was introduced as a novel way to conceptualise psychopathology in general, and depression in particular. ${ }^{6}$ It has been argued that the traditional medical disease model in which an underlying disorder, such as a lung tumour, causes specific symptoms (such as chest pain) is inappropriate for mental disorders. Within the network perspective, depressive symptoms such as fatigue or anhedonia are not merely seen as elements of an overarching disease called depression, but as separate symptoms that are related to each other. It moves away from the traditional assumption that observable (depressive) symptoms are caused by a latent, unidimensional variable (e.g. depression). ${ }^{6}$

Instead, a typical network consists of symptoms as the nodes and their mutual associations as the edges in the network. Such a network enables the analysis of how a symptom, such as fatigue, is connected with other symptoms, for example anhedonia. ${ }^{6}$ symptom: if a symptom (e.g. fatigue) has many and/or strong associations to other symptoms, they are more central within the network than a less connected symptom. ${ }^{6,7}$ There are different mechanisms represented by centrality such as strength, betweenness and closeness. Independently of the underlying mechanisms, central symptoms are believed to have considerable impact on other symptoms and, consequently, more strongly predict the course or onset of psychopathology than symptoms that are less central. ${ }^{6,7}$ For example, a study predicting the future course of depression showed that the most interconnected or central depressive symptoms (in this case fatigue and depressed mood) at baseline were the most predictive of future depression. ${ }^{8}$

Networks can be analysed on their own or compared with other networks. On this note, van Borkulo et al ${ }^{9}$ found that the baseline depressive symptom networks of patients with major depressive disorder were related to the course of depression, that is, a more densely connected network at baseline was associated with the persistence of depression over time.

The network perspective also influences the manner in which we assess and analyse outcomes in clinical studies. ${ }^{10}$ In most studies, the level of depression is estimated by simply adding up scores of different depressive symptoms to create an aggregate score. According to network analyses, typical depressive symptoms such as sad mood, insomnia or loss of appetite are distinct phenomena, and not merely elements of one overall construct of depression. The use of (change in) aggregate scores to estimate the effectiveness of interventions is argued to be part of the reason why our understanding of depression and its aetiology is limited. ${ }^{10}$ 


\section{Applying network analysis in suicide research}

Network analysis has been applied in the field of depression, post-traumatic stress and psychosis research, but not yet in the field of suicidology. Given the complexity of suicidal behaviour, network analysis may offer new insights that have been previously unavailable. ${ }^{11}$ When assessing suicidality, the most commonly used scale is the Beck Scale for Suicide Ideation (SSI). ${ }^{12}$ The SSI consists of 19 items, assessing thoughts and actions, and has been (for the most part) found to be unidimensional, that is, all items measure one construct, usually labelled suicidality. ${ }^{13,14}$ Traditionally, the separate items of the SSI are thought to be caused by suicidality, and not the other way around (Fig. 1). The level of suicidality is determined by summing up the scores on the 19 individual items. However, the total score lacks predictive validity, making it a less useful instrument for clinical studies and treatment settings. ${ }^{14}$

A recent study using item response analysis showed which items of the SSI were more important than others when assessing level of suicidality. ${ }^{15}$ The item 'desire for an active attempt' differentiated best between patients with high and low risk. However, using item response analysis, it is not possible to demonstrate the complex relationship between the separate items.

In this study, for the first time, we applied network analysis to examine the complex relationship between the 19 suicidal symptoms as assessed by the SSI and future suicidal behaviour within a sample of patients who were admitted to hospital following a suicide attempt.

Network analysis allows us to understand which suicide symptoms are most central in comparison with the other suicide symptoms. We expect that some, but not all, symptoms are directly related to future suicidal behaviour. More specifically, in line with the integrated motivational-volitional (IMV) model $^{4}{ }^{4}$ we expect the more action-related variables (the volitional-phase factors) of the SSI to be directly related to suicidal behaviour, when compared with more contemplative factors (the motivational-phase factors). Additionally, similar to van Borkulo et al, we hypothesised that suicidal symptoms will be more densely connected at baseline in patients who engaged in repeat suicidal behaviour within 15 months of their index admission to hospital when compared with patients that did not reattempt at follow-up.

\section{Method}

\section{Participants}

We analysed data from a prospective study of psychological predictors of repeat suicidal behaviour in a sample of patients who were admitted to a single general hospital in Edinburgh, Scotland, following a suicide attempt. ${ }^{16}$ From January 2008 until September 2009, 432 patients assessed by the liaison psychiatry service the day after presenting to a single general hospital following a suicide

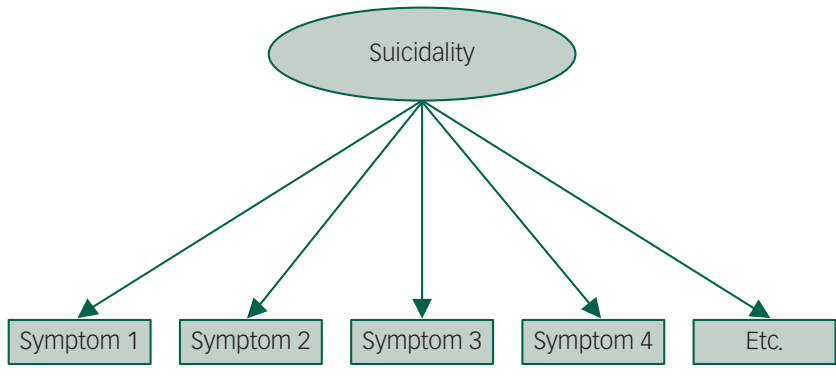

Fig. 1 The relationship between suicidality and the observable symptoms. According to the traditional medical modelling, suicidality is the root cause of the underlying symptoms. attempt were invited to participate in the study. At baseline, 388 patients were asked directly by a member of the research team whether they had intended to end their lives and they were only included in the sample if they confirmed this to be the case. As this study presents a secondary analysis of previously collected data, no additional ethical approval was sought as the network analyses are consistent with the original study aims.

\section{Beck Scale for Suicide Ideation}

Suicidal symptoms were assessed using the SSI. ${ }^{12}$ This scale contains 19 items assessing suicidal thoughts and plans, and two additional items that cover the number of previous attempts (zero, one and two or more) and suicidal intent during the previous attempts. The total score is determined by summing the first 19 items. Each item is rated on a 3-point scale from 0 to 2, a higher score indicating a higher level of suicidality. Scores range from 0 to 38. Scores on the SSI are normally skewed, in that most patients often have a low score, and only a few have a score of two or higher. ${ }^{13}$ However, in our high-risk sample, the scores are likely to be normally distributed. Also, network analyses do not depend on the psychometrics of the scale, because they deal with bi-partial associations between variables.

To make the network visualisation more parsimonious to interpret, two of the authors separately coded the items of the SSI into more action-related (volitional phase) and more motivational-phase symptoms based on content of the items according to the IMV model $^{4}$ (Table 1). The IMV model is a predominant theoretical model developed to understand the factors that lead to suicidal behaviour. It builds on empirical and conceptual evidence from other models, and the evidence for its validity is growing. ${ }^{15}$ We used the network analysis to test the basic premise of the IMV model, namely that volitional factors are more strongly related to repeat suicidal behaviour than motivational factors.

Items $1-8$ and item 11 were identified as motivational-phase symptoms (e.g. wish to die). Items 9, 10 and 12-19 were deemed to measure volitional-phase symptoms, including items such as control over action. Authors agreed on all items. It is important to note that the classification of the items into motivational- and volitional-phase factors does not influence the algorithms of the networks analyses.

\section{Repeat suicidal behaviour at follow-up}

Using a national linkage database, it was determined whether a patient had been admitted to any Scottish hospital within 15 months of the index episode following self-harm. The Information Services Division of the Scottish National Health Service was able to link $96.4 \%$ of the initial sample (374/388). In addition, two trained coders independently examined extracts from the medical records to determine the presence or absence of suicide intent associated with any episodes of self-harm. They agreed that for 94 out of the 101 patients who were admitted for self-harm, there was presence of suicide intent. So, in the original study, data were used for 367 patients who were linked and for whom the researchers were able to determine suicide intent if they were readmitted to the hospital for self-harm during the follow-up.

\section{Data analysis \\ General analysis}

For each item of the SSI, we present the mean (s.d.) for the total group, as well as the means (s.d.) for repeaters and non-repeaters separately. To test differences on each item of the SSI between repeaters and non-repeaters, we performed a Wilcoxon rank sum test for ordinal data. ${ }^{9} P$-values and effect sizes are also presented. 
Table 1 Analysis of item scores for the total group, and for repeaters and non-repeaters

\begin{tabular}{|c|c|c|c|c|c|}
\hline Symptom (abbreviation) & $\begin{array}{l}\text { Total group ( }(n=366) \\
\text { Mean (s.d.) }\end{array}$ & $\begin{array}{l}\text { Repeaters }(n=94) \\
\text { Mean (s.d.) }\end{array}$ & $\begin{array}{c}\text { Non-repeaters }(n=272) \\
\text { Mean (s.d.) }\end{array}$ & P & Effect size \\
\hline \multicolumn{6}{|l|}{ Motivational-phase symptoms } \\
\hline Wish to live (liv) & $1.16(0.71)$ & $1.30(0.67)$ & $1.11(0.72)$ & $0.03^{*}$ & 0.6 \\
\hline Wish to die (die) & $1.37(0.74)$ & $1.51(0.67)$ & $1.32(0.78)$ & $0.03^{*}$ & 0.7 \\
\hline Reasons for living/dying (rea) & $1.20(0.79)$ & $1.37(0.73)$ & $1.14(0.81)$ & $0.01^{*}$ & 0.6 \\
\hline Desire for active attempt (des) & $1.31(0.75)$ & $1.57(0.60)$ & $1.22(0.78)$ & $<0.001^{* * *}$ & 0.7 \\
\hline Passive desire (pas) & $1.01(0.80)$ & $1.20(0.76)$ & $0.94(0.80)$ & $0.007^{* *}$ & 0.5 \\
\hline Duration of suicide ideation (dur) & $0.99(0.77)$ & $1.15(0.70)$ & $0.93(0.79)$ & $0.02^{*}$ & 0.5 \\
\hline Frequency of suicide ideation (freq) & $0.89(0.72)$ & $1.11(0.66)$ & $0.82(0.72)$ & $<0.001 * * *$ & 0.5 \\
\hline Attitude suicidal behaviour (att) & $1.15(0.76)$ & $1.37(0.69)$ & $1.08(0.77)$ & $0.001^{* *}$ & 0.6 \\
\hline Cry for help versus cry for pain (cry) & $1.42(0.79)$ & $1.56(0.63)$ & $1.38(0.83)$ & 0.14 & 0.7 \\
\hline \multicolumn{6}{|l|}{ Volitional-phase symptoms } \\
\hline Control over action (con) & $0.86(0.68)$ & $1.12(0.67)$ & $0.78(0.66)$ & $<0.001^{* * *}$ & 0.5 \\
\hline Deterrents of attempt (det) & $1.01(0.75)$ & $1.28(0.69)$ & $0.92(0.74)$ & $<0.001^{\star * *}$ & 0.5 \\
\hline Actual planning (pla) & $0.90(0.79)$ & $1.16(0.74)$ & $0.81(0.79)$ & $<0.001^{* * *}$ & 0.4 \\
\hline Availability of methods (met) & $1.18(0.89)$ & $1.38(0.80)$ & $1.11(0.91)$ & $0.01^{*}$ & 0.5 \\
\hline Courage for actual behaviour (cou) & $1.83(0.76)$ & $1.31(0.66)$ & $1.14(0.79)$ & $0.01^{*}$ & 0.6 \\
\hline Expectancy of actual attempt (exp) & $1.04(0.77)$ & $1.30(0.65)$ & $0.95(0.79)$ & $<0.001^{\star *}$ & 0.5 \\
\hline Actual preparation (pre) & $0.61(0.73)$ & $0.80(0.82)$ & $0.55(0.69)$ & $0.01^{*}$ & 0.2 \\
\hline Suicide note (not) & $0.66(0.83)$ & $0.71(0.80)$ & $0.64(0.84)$ & 0.30 & 0.2 \\
\hline Arrangements after death (arr) & $0.37(0.63)$ & $0.52(0.73)$ & $0.31(0.59)$ & $0.008^{* *}$ & 0.0 \\
\hline Concealment about ideation (cea) & $1.0(0.85)$ & $1.15(0.82)$ & $0.94(0.85)$ & $0.048^{*}$ & 0.5 \\
\hline
\end{tabular}

The network structure of suicide symptoms and repeat suicidal behaviour

We estimated the relationship between the 19 suicidal symptoms. Both the visualisation of the network and the centrality measures are presented in the results section. This analysis allows us to understand which suicidal symptoms are most central compared with the other suicidal symptoms. Next, we estimated the relationship between suicidal symptoms and repeat suicidal behaviour, by constructing a network using both the SSI items and the dichotomous outcome variable that indicated whether a patient engaged in repeat suicidal behaviour in the following 15 months or not. Including this outcome variable allowed us to investigate which suicidal symptoms are prominently related to repeat suicidal behaviour.

Conceptually, a network is a visualisation of the associations (correlation) between symptoms. The start of every network analysis is therefore a matrix consisting of the correlations between relevant variables. We used the cor_auto function in $R$ to compute the appropriate correlation matrix given the ordinal structure of the items of the SSI. However, in psychiatry, many spurious (indirect) relationships exist. We therefore used a partial correlation matrix, which controls for spurious relationships. Finally, a so-called L1 penalty is imposed on the partial correlations ${ }^{17,18}$ to obtain the optimal balance between sparsity of the model and goodness of fit. ${ }^{19}$ Moreover, this procedure circumvents multiple testing problems that occur with conventional hypothesis testing of partial correlations - in our case this would require $171(19 \times 18 / 2)$ tests. L1 regularisation depends on a socalled hyperparameter $\gamma$, which we set to $0.5 .^{20}$ Assuming that a set of sparse pairwise relations underlie the data, this procedure converges to the true network. ${ }^{21,22}$ To estimate the L1 regularised partial correlations network structure, we used $\mathrm{R}$ package qgraph. ${ }^{20}$

\section{Network visualisation}

To visualise the different networks, we also used the $\mathrm{R}$ package qgraph. ${ }^{20}$ To generate a visualisation that shows how symptoms cluster, we used the Fruchterman-Reingold algorithm. ${ }^{22}$ This algorithm results in a visualisation in which nodes with more and/or stronger connections are placed closer to each other.

\section{Measures of node centrality}

We present three estimates of centrality to investigate the relative importance of the 19 symptoms of the suicide symptom network: strength, closeness and betweenness. ${ }^{23-25}$ The strength of a node is calculated by summing the magnitude of each of its edges. Closeness is inversely proportional to the mean shortest distance from the node to all other nodes in the network. The betweenness index indicates the number of times that a node lies on the shortest paths between other nodes. In addition, all three measures indicate a different level of centrality of a symptom: the higher the value, the higher the centrality. We standardised the centrality measures so they can be more easily compared with each other.

\section{Testing differences in network structure of repeaters and non-} repeaters

To test the final hypothesis - whether repeaters have a more densely connected network of suicidal symptoms at baseline than non-repeaters - we compared the density of the baseline structure (global strength) of the 19 symptoms of the SSI for both groups, that is, non-repeaters and repeaters. The global strength can be defined as the weighted sum of absolute connections, ${ }^{26}$ and it was determined for both repeaters and non-repeaters. To test for any significant differences in global network strength between the two groups, we used the Network Comparison Test (NCT), which reliably tests for differences in network structures of two groups. ${ }^{27}$ NCT is a two-tailed permutation test in which the difference in network structure of the two groups - in our case repeaters and non-repeaters - is calculated repeatedly (1000 times) for randomly regrouped individuals. This test results in a reference distribution: a distribution under the null hypothesis that both groups are equal. The reference distribution can be used to test the observed difference in network structure of the two groups (at the threshold of 0.05). R package NCT was used. ${ }^{27}$ 


\section{Results}

Of the 367 patients, we used data for 366 patients (158 males, mean age 35 years (s.d.=13.6)) who had $<5$ missing values on the SSI. Among these 366 patients, 6 had 2 values missing, and 5 had 1 value missing. We used the patients' mean item scores and rounded up to a whole number to impute these missing data (4). Total scores on the SSI ranged from 0 to 38 , and the mean score was 19 (s.d.=10.3) and a median of 21. At follow-up, 94 patients (44 males and 50 females) were treated in hospital following a repeat suicide attempt. No significant differences were found between repeaters and non-repeaters in terms of age.

Repeaters scored significantly higher on all items of the SSI except for the items 'cry for help versus cry for pain' (higher score would have indicated a cry for pain, which is argued to be related to a higher risk of suicidal behaviour ${ }^{12}$ ) and 'suicide note' compared with the non-repeaters (Table 1). Effect sizes ranged from 0.0 (negligible) to 0.7 (large). Wish to die, desire for an active attempt and cry for help $v$. cry for pain had the largest effect size (0.7). Of the 94 repeaters, $74(80 \%)$ indicated at baseline that they had attempted suicide two or more times before their index attempt. Eighteen patients had attempted once before, and for one patient, this was their first attempt. Within the group of nonrepeaters, 157 (57\%) patients had attempted suicide two or more times before the initial admission to hospital. A total of 97 patients stated that they had made one previous attempt, and for 18 patients this was the first attempt.

\section{Network analyses of suicide symptoms}

When visualising the network of the 19 symptoms of the SSI, a few observations are noteworthy (Fig. 2(a)). For one, actionrelated volitional-phase symptoms tend to cluster, as do motivational-phase symptoms. When looking at the centrality of the symptoms, we find that desire for an active attempt (a motivational-phase symptom) had the highest score on all three centrality indices (Fig. 2(b)). Other central symptoms were expectancy of an actual attempt (volitional-phase symptom) and attitude towards suicide (motivational-phase symptom).

(a)

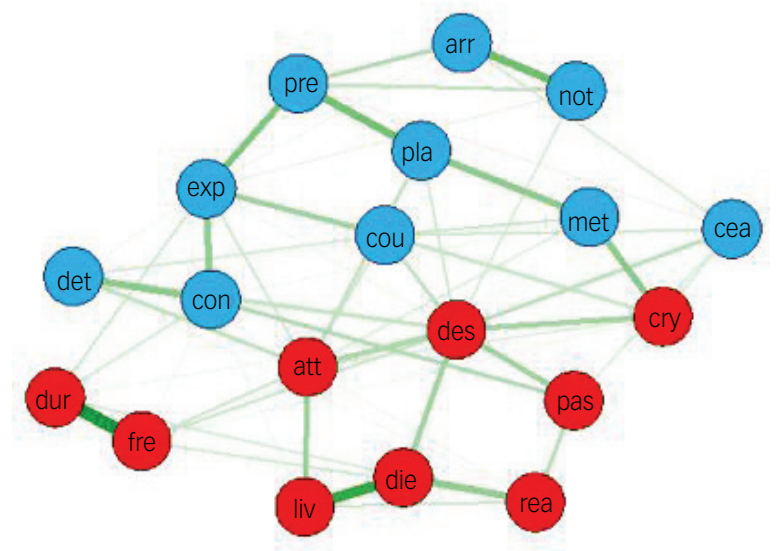

\section{Network analyses of suicide symptoms and repeat suicidal behaviour}

In Fig. 3, we added the outcome variable repeat suicidal behaviour ( 0 for no attempt and 1 for attempt at follow-up) to the network of suicide symptoms illustrated in Fig. 2. Both the network visualisation (Fig. 3) and the partial correlations matrix (online appendix) showed that seven volitional-phase (action-related) symptoms (i.e. control over action, deterrents of an attempt, actual planning, courage for actual suicidal behaviour, expectancy of an actual attempt, suicide note and arrangements after death) and three motivational symptoms (the desire for an active attempt, wish to die and cry for pain $v$. cry for help) were directly related to repeat suicidal behaviour. The centrality measures were comparable with the outcomes of Fig. 2(b), with the desire for an active attempt being the most central item.

\section{Differences in baseline structure between repeaters and non-repeaters}

In Fig. 4(a) and (b), we visualised the network structure of repeaters and non-repeaters. The network of repeaters had a total of 82 connections: 73 positive and 9 negative connections. The non-repeater network found 87 connections: 82 positive and 5 negative connections. NCT revealed that this difference was not significant $(P=0.16)$. Testing the difference in density when including only the 10 symptoms that were found to be directly related to repeat suicidal behaviour in Fig. 3, again, yielded no significant differences $(P=0.15)$.

\section{Centrality for repeaters and non-repeaters}

When comparing the centrality indices, comparable patterns for repeaters and non-repeaters were found (Fig. 5). As for the total sample, desire for an active attempt had the highest score on all indices for both groups.

\section{Sensitivity analysis}

Similar results were found when using the raw data, so without mean imputation.

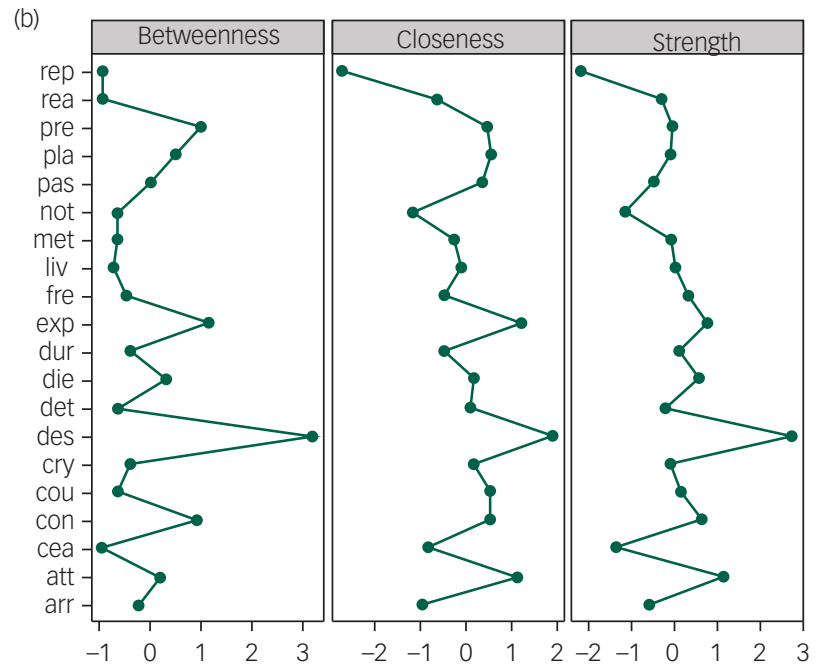

Fig. 2 (a) Network visualisation of suicide symptoms at baseline. (b) Centrality indices of symptoms.

Blue nodes present volitional-phase symptoms and red nodes present motivational-phase symptoms. Green connections present positive associations. Thicker edges present stronger associations. arr, arrangements after death; att, attitude towards suicidal behaviour; cea, concealment about ideation; con, control over action; cou, courage for actual behaviour; cry, cry for help versus cry for pain; des, desire for active attempt; det, deterrents of attempt; die, wish to die; dur, duration of suicide ideation; exp, expectancy of actual attempt; fre, frequency of suicide ideation; liv, wish to live; met, availability of methods; not, suicide note; pas, passive desire; pla, actual planning; pre, actual preparation; rea, reasons for living dying. 


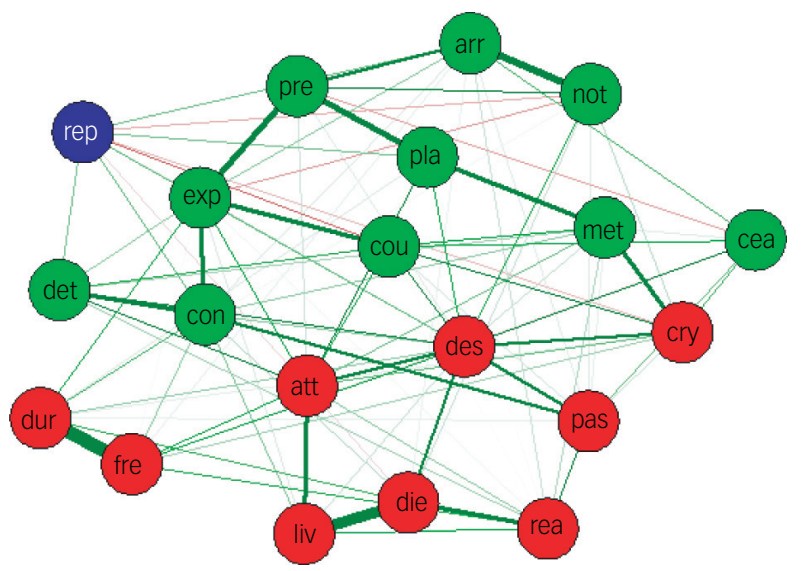

Fig. 3 A network visualisation of suicide symptoms at baseline and repeat suicidal behaviour within 15 months.

Green nodes present volitional-phase symptoms and red nodes present motivational-phase symptoms. Green connections present positive associations. The blue node represents suicidal behaviour at 15 months' follow-up. Thicker edges present stronger associations. arr, arrangements after death; att, attitude towards suicidal behaviour; cea, concealment about ideation; con, control over action; cou, courage for actual behaviour; cry, cry for help versus cry for pain; des, desire for active attempt; det, deterrents of attempt; die, wish to die; dur, duration of suicide ideation; exp, expectancy of actual attempt; fre, frequency of suicide ideation; liv, wish to live; met, availability of methods; not, suicide note; pas, passive desire; pla, actual planning; pre, actual preparation; rea, reasons for living dying; rep; repeat suicidal attempt.

\section{Discussion}

In this paper, for the first time, we have applied the network approach to suicidal behaviour to better understand the complex relationship between suicidal symptoms, and between suicidal symptoms and repeat suicidal behaviour. The analysis revealed that the desire for an active attempt was the most central symptom (i.e. had the most or strongest association with other symptoms), followed by the expectancies about an attempt and the attitude towards suicide. When extending the network with the outcome variable suicidal behaviour at 15 -month follow-up, we found that not all symptoms were directly related to future suicidal behaviour. More specifically, we found seven volitional-phase (action-related) symptoms and three motivational-phase symptoms to be directly related to repeat suicidal behaviour. When comparing the results from the network analysis with the traditional Wilcoxon analysis (Table 1), we find that network analysis offers a much more detailed insight in the complex relationship between the 19 variables and repeat suicidal behaviour.

We also hypothesised that the network of symptoms at baseline would be more densely connected for repeat attempters than for non-repeaters. As no significant difference in network density between the two groups was found, this hypothesis was not supported. Our null finding might be explained by the fact that as all patients within our sample had survived a suicide attempt, and therefore, all had an elevated risk for future suicidal behaviour, the networks of repeaters very much resembled the network of non-repeaters. Comparing the network density between suicide ideators and suicide attempters might be a better method to relate network density to future suicidal behaviour. The lack of significance can also be explained by the relative small number $(n=94)$ of repeaters within our sample. As a consequence, estimating the relationship between 19 nodes with 94 patients who repeated may have resulted in an unreliable network structure. ${ }^{21}$ Moreover, the difference in sample size could have resulted in a loss of power to detect differences by NCT. ${ }^{26}$

It is interesting to compare the results of the network analysis with the results of an item response-based analysis of the SSI. ${ }^{23}$ Although conducted within a different sample, the item response analysis also found the 'desire for an active attempt' to be the most informative item. Another central item in the network analysis, the 'expectancy of an active attempt' also showed high discriminatory value within the item response analysis. What a network analysis adds to an item response analysis is that it visualises the complex relation between the different symptoms and repeat suicidal behaviour. Also, item response analysis is only
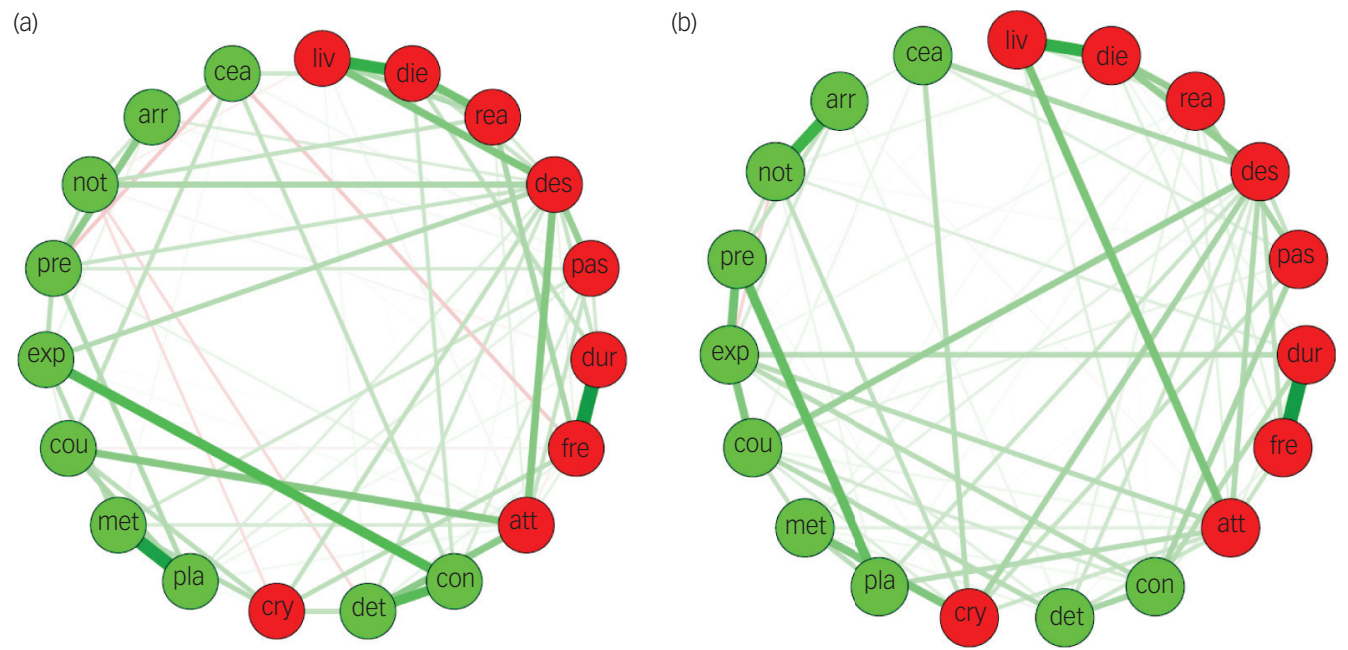

Fig. 4 (a) Network structure of repeaters $(n=94)$. (b) Network structure of non-repeaters $(n=272)$.

Green nodes present volitional-phase symptoms and red nodes present motivational-phase symptoms. Green connections present positive associations, red connections present negative associations. Thicker edges present stronger associations (positive and negative). arr, arrangements after death; att, attitude towards suicidal behaviour; cea, concealment about ideation; con, control over action; cou, courage for actual behaviour; cry, cry for pain versus cry for help; des, desire for active attempt; det, deterrents of attempt; die, wish to die; dur, duration of suicide ideation; exp, expectancy of actual attempt; fre, frequency of suicide ideation; liv, wish to live; met, availability of methods; not, suicide note; pas, passive desire; pla, actual planning; pre, actual preparation; rea, reasons for living dying. 


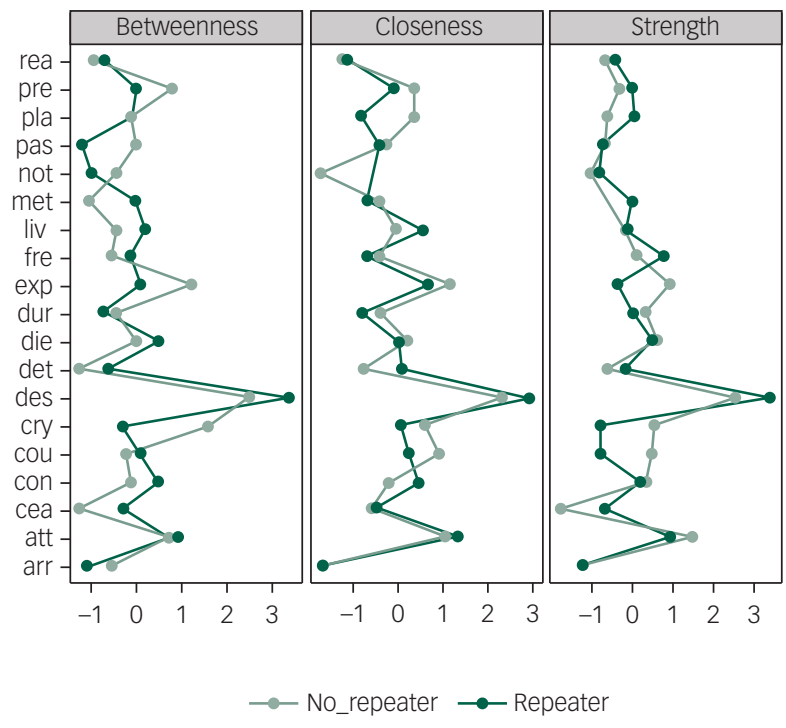

Fig. 5 Node centrality measures for repeaters and non-repeaters: betweenness, closeness and strength.

arr, arrangements after death; att, attitude towards suicidal behaviour; cea, concealment about ideation; con, control over action; cou, courage for actual behaviour; cry, cry for pain versus cry for help des, desire for active attempt; det, deterrents of attempt; die, wish to die; dur, duration of suicide ideation; exp, expectancy of actual attempt; fre, frequency of suicide ideation; liv, wish to live; met, availability of methods; not, suicide note; pas, passive desire; pla actual planning; pre, actual preparation; rea, reasons for living dying.

possible under strict assumptions such as unidimensionality of the scale, ${ }^{23}$ whereas network analysis is not dependent on the psychometric properties of a scale, as it is based on the association between separate items.

As all networks showed that the desire for an attempt is the most central symptom, this could be the starting point for new therapeutic interventions. Any improvement in this symptom is likely to influence other symptoms and possibly influence repeat suicidal behaviour. Indeed, when inspecting the different networks, it might be argued that the first threshold for a suicide attempt is an active desire, and the second threshold is related to expectancy for an actual attempt and loss of control. Although we have no information about directionality or causality, the network approach can help clinicians to think about the relationship with specific suicidal symptoms and repeat suicidal behaviour, and to inspire future research on this topic aimed at unravelling the symptom network structure. In this study, we found that both motivational- and volitional-phase symptoms play a central role in the symptomatology of suicidal patients. Consistent with the IMV model, ${ }^{4,16}$ volitional-phase symptoms are more directly related to predicting suicidal behaviour. This might indicate that when assessing or treating patients after they have been treated for their suicide attempt, both sets of symptoms warrant attention. In an acute setting, however, focusing on volitional-phase symptoms is likely to have a greater impact on reducing risk of repeat suicidal behaviour. Indeed, a recent exploratory study found that targeting volitional-phase symptoms via a volitional help sheet yielded promise in reducing future suicidal ideation and behaviour. ${ }^{28}$ The concept of a network of symptoms can also be used during treatment as a form of psychoeducation, to explain to the patient how suicidal behaviour can develop. Finally, in The Netherlands, an innovative study is starting that allows patients to collect such data via their smartphone. ${ }^{29}$ The aim of this study is to provide patients with an individual network of relevant symptoms that can be shared with the therapist on a weekly basis, allowing more tailored treatment possible.

\section{Strengths and limitations}

This is a completely novel approach to investigate the association between suicidal symptoms and suicidal behaviour over time. A key strength of this study is the application of a state-of-the-art statistical technique (network analysis) to one of the most widely used scales to assess suicidality (the SSI).

An important limitation is worthy of note. As all of the symptoms data were collected at a single time point, we cannot investigate dynamic interactions, which would require intensive longitudinal data, collected via mobile telephones and employing ecological momentary assessment techniques. ${ }^{5,10}$ Only when a network is based on longitudinal data, the associations in the network are temporal (i.e. one symptom at one time point has an effect on another symptom the next time point), whereas when the network is based on cross-sectional data, the associations in the network are not temporal (i.e. there is no directionality). However, we can investigate how symptoms co-occur and are related to each other, which helps our understanding of suicidality and offers directions for future studies.

Also, it has yet to be demonstrated whether targeting relevant central symptoms will actually benefit patients. There have yet been no studies in which the effectiveness of targeting central symptoms have been tested. ${ }^{7}$ The previously mentioned Dutch smartphone-based study is expected to offer first insights of the applicability of individual networks and centrality analysis during therapy. Results are expected around 2019. ${ }^{29}$

In addition, although the SSI is the most widely used scale for suicidal thoughts and behaviour, it has many flaws, such as having an inadequate 3 -point response set. ${ }^{25}$ Other weaknesses in the SSI were identified by de Beurs et al. ${ }^{13}$ Operationalising the outcome variable is also not without its challenges; indeed, a recorded suicide attempt resulting in a hospital presentation is far from perfect. Suicide attempt status, $v$. no attempt, is arguably a false dichotomy, as we are likely to have missed, for example, suicide attempts that did not need hospital treatment. Several studies have provided evidence that suicidality exists along a spectrum and that suicidal behaviours are best understood through the individual's degree of intent to die associated with the behaviour (see e.g. De Leo et al $^{30}$ ). It is probable that many of the 'non-repeaters' did experience strong suicidal feelings and thoughts. It is also probable that some of the 'repeat attempters' had varying degrees of suicidal intent associated with their actions. In addition, it is also likely that some of the 'nonattempters' made serious attempts after the study data collection period, and possibly even died by suicide.

The SSI items were not designed to measure motivational- or volitional-phase symptoms, making the assessment of the two factors suboptimal. However, as the overarching aims of this study were to demonstrate the utility of network analysis in suicide research and to demonstrate how networks between subgroups can be compared, the present findings represent an important advance in knowledge. Nonetheless, future network analysis should endeavour to use other scales, ideally scales that assess a more extensive range of suicidal symptoms and that aim to assess suicide intent associated with all forms of suicidal behaviour (i.e. both hospital-treated and non-hospital-treated suicidal behaviour).

Finally, the difference between the networks of patients who had made an attempt for the first time, patients who had made an earlier attempt and patients who had already made two or more attempts would be of great clinical interest. Much larger sample sizes are needed for these types of analyses. These analyses will be done in the 
near future on a large multiyear cohort of hospital-treated suicide attempters from Belgium. ${ }^{31}$ Baseline data of 15000 patients is available, making subgroup analysis much more feasible.

In short, this study presents important new insights into how network analysis can be used to advance our understanding and prediction of suicidal behaviour. As network analysis has motivated work on other psychiatric disorders such as depression ${ }^{6-9}$ and psychosis, ${ }^{32}$ this study aims to stimulate the use of network analysis in suicide research. Many papers on network analysis use previously collected data to perform secondary analysis. In most cases, additional ethical approval will not be necessary, making the secondary analysis of existing data a feasible starting point for network analysis within suicide research. The $\mathrm{R}$ code of this article is freely available via the first author, and many tutorials are available via http://psychosystems.org/.

Derek P. de Beurs, PhD, Netherlands Institute for Health Services Research (NIVEL) Utrecht, The Netherlands; Claudia D. van Borkulo, PhD, Department of Psychology, Psychological Methods Group, University of Amsterdam, The Netherlands; Rory C. O'Connor, PhD, Suicidal Behaviour Research Laboratory, Institute of Health \& Wellbeing, University of Glasgow, Glasgow, UK

Correspondence: Derek P. de Beurs, Netherlands Institute for Health Services Research (NIVEL), The Netherlands. Email: d.debeurs@nivel.nl

First received 6 Nov 2016, final revision 28 Mar 2017, accepted 29 Mar 2017

\section{Funding}

The initial study of R.C.O'C. was supported by funding from the Chief Scientist Office, Scottis Government (CZH/4/449). For the additional network analysis, no additional funding was obtained.

\section{References}

1 World Health Organization. Preventing Suicide: A Global Imperative. WHO, 2014.

2 Hawton K, van Heeringen K. Suicide. Lancet 2009; 373: 1372-81.

3 O'Connor RC, Nock MK. The psychology of suicidal behaviour. Lancet Psychiatry 2014; 1: 73-85.

4 O'Connor RC. Towards an integrated motivational-volitional model of suicida behaviour. In International Handbook of Suicide Prevention: Research, Policy and Practice (eds RC O'Connor, S Platt, J Gordon): 181-98. Wiley-Blackwell, 2011.

5 de Beurs D, Kirtley O, Kerkhof A, Portzky G, O'Connor RC. The role of mobile phone technology in understanding and preventing suicidal behavior (editorial). Crisis 2015; 36: 79-82.

6 Borsboom D, Cramer AO. Network analysis: an integrative approach to the structure of psychopathology. Annu Rev Clin Psychol 2013; 9: 91-121.

7 Fried El, van Borkulo C, Cramer AOJ, Boschloo L, Schoevers RA, Borsboom D. Mental disorders as a network of problems: a review of recent insights. Soc Psychiatry Psychiatr Epidemiol 2017; 52: 1-10.

8 Boschloo L, van Borkulo C, Borsboom D, Schoevers RA. A prospective study on how symptoms in a network predict the onset of depression. Psychother Psychosom 2016; 85: 183-4.

9 van Borkulo C, Boschloo L, Borsboom D, Penninx BW, Waldorp LJ, Schoevers RA. Association of symptom network structure with the course of longitudina depression. JAMA Psychiatry 2015; 72: 1219-26.

10 Fried EL, Nesse RM. Depression sum-scores don't add up: why analyzing specific depression symptoms is essential. BMC Med 2015; 13: 72
11 de Beurs D. Network analysis: a novel approach to understand suicidal behaviour Int J Environ Res Public Health 2017; 14: 219

12 Beck AT, Kovacs M, Weissman A. Assessment of suicidal intention: the Scale for Suicide Ideation. J Consult Clin Psychol 1979; 47: 343-52

13 de Beurs DP, de Vries ALM, de Groot MH, de Keijser J, Kerkhof AJFM. Applying computer adaptive testing to optimize the assessment of suicidal behavior: a simulation study. J Med Internet Res 2014; 16: e207.

14 de Beurs DP, Fokkema M, O'Connor RC. Optimizing the assessment of suicidal behavior: the application of curtailment techniques. J Affect Disord 2016; 196 218-24

15 O'Connor RC, Cleare S, Eschle S, Wetherall K, Kirtley OJ. The integrated motivational-volitional model of suicidal behavior. In International Handbook of Suicide Prevention (eds RC O'Connor, J Pirkis): 220-40. Wiley, 2016.

16 O'Connor RC, Smyth R, Williams JMG. Intrapersonal positive future thinking predicts repeat suicide attempts in hospital-treated suicide attempters. I Consult Clin Psychol 2015; 83: 169-76

17 Friedman J, Hastie T, Tibshirani R. Sparse inverse covariance estimation with the graphical lasso. Biostatistics 2008; 9: 432-41.

18 Tibshirani R. Regression shrinkage and selection via the lasso. J R Stat Soc Series B Stat Methodol 1996; 58: 267-88.

19 Costantini G, Epskamp S, Borsboom D, Perugini M, Mõttus R, Waldorp L, et al. State of the aRt personality research: a tutorial on network analysis of personality data in R. J Res Pers 2015; 54: 13-29.

20 Epskamp S, Cramer AO, Waldorp LJ, Schmittmann VD, Borsboom D. Qgraph: network visualizations of relationships in psychometric data. J Stat Softw 2012; 48: 1-18.

21 van Borkulo CD, Borsboom D, Epskamp S, Blanken TF, Boschloo L, Schoevers RA et al. A new method for constructing networks from binary data. Sci Rep 2014 4: 5918

22 Fruchterman TM, Reingold EM. Graph drawing by force-directed placement. Softw Pract Exp 1991; 21: 1129-64.

23 Barrat A, Barthelemy $M$, Pastor-Satorras $R$, Vespignani $A$. The architecture of complex weighted networks. Proc Natl Acad Sci U S A 2004; 101: 3747-52.

24 Boccaletti S, Latora $V$, Moreno $Y$, Chavez M, Hwang D-U. Complex networks: structure and dynamics. Phys Rep 2006; 424: 175-308.

25 Opsahl T, Agneessens F, Skvoretz J. Node centrality in weighted networks: generalizing degree and shortest paths. Soc Netw 2010; 32: 245-51.

26 Bonacich P. Power and centrality: a family of measures. Am J SOC 1987; 92 $1170-82$.

27 Van Borkulo CD. Network Comparison Test. 29 October 2016. Available at https:// cran.r-project.org/web/packages/NetworkComparisonTest/NetworkComparisonTest.pdf

28 Armitage CJ, Rahim WA, Rowe R, O'Connor RC. An exploratory randomised trial of a simple, brief psychological intervention to reduce subsequent suicidal ideation and behaviour in patients admitted to hospital for self-harm. Br J Psychiatry 2016; 5: $470-6$.

29 Nuij N, van Ballegooijen W, Ruwaard J, de Beurs DP, O'Connor RC, Smit JH, et al. Smartphone-based safety planning and self-monitoring for suicidal patients: a conceptual paper of the CASPAR (Continuous Assessment for Suicide Prevention and Research) study. Preprints 2017, 2017040103 (doi: 10.20944/preprints201704. 0103.v1).

30 De Leo D, Burgis S, Bertolote JM, Kerkhof AJFM. Definitions of suicidal behavior Lessons learning from the WHO/EURO Multicentre study. Crisis 2016; 27: 4-15.

31 Vancayseele N, Portzky G, van Heeringen $\mathrm{K}$. Increase in self-injury as a method of self-harm in Ghent, Belgium: 1987-2013. PLOS One 2016; 11: e0156711.

32 Wigman JTW, de Vos S, Wichers M, van Os J, Bartels-Velthuis AA. A transdiagnostic network approach to psychosis. Schizophr Bull 2017; 43: 122-32. 